\title{
NUMERICAL AND EXPERIMENTAL INVESTIGATION OF THE FLOW FIELD AROUND FINITE CYLINDERS PLACED ON A FLAT WALL: THE EFFECT OF FREE-END SHAPE \\ E.M.Wahba and Samy M. El-Behery \\ Mechanical Power Engineering Department, \\ Faculty of Engineering, Menoufia University, Shebin El-Kom, Egypt.
}

\begin{abstract}
Numerical and experimental study of the three-dimensional unsteady flow field around finite cylinders placed on a ground wall is presented. The effects of free-end shape reading flat, hemisphere and cone top of the circular cylinder are examined. The numerical results were obtained using the computational fluid dynamic (CFD) code, Fluent 6.3.26. The three-dimensional unsteady flow filed is investigated using unsteady Reynolds averaged Navier-Stokes (URANS) equation along with the four equations $v^{2}-f$ turbulence model. This turbulence model is implemented in the CFD code using User Defined Function (UDF) and User Defined Scalars (UDS). The measurements of surface pressure distribution around the bodies are presented. The cylinder has an aspect ratio of $1.33\left(\mathrm{~h} / \mathrm{D}=1.33\right.$ ) and faces flow with Reynolds number of $1.7 \times 10^{5}$ based on free stream velocity and cylinder diameter. The results show that the top free surface of the models affects the flow field, such as the development of the wake structure, recirculation zone and the reattachment zone. The results indicate also that the hemispherical free end cylinder is subjected to the larger flow induced vibration while the cone free end is subjected to the lowest flow induced vibration

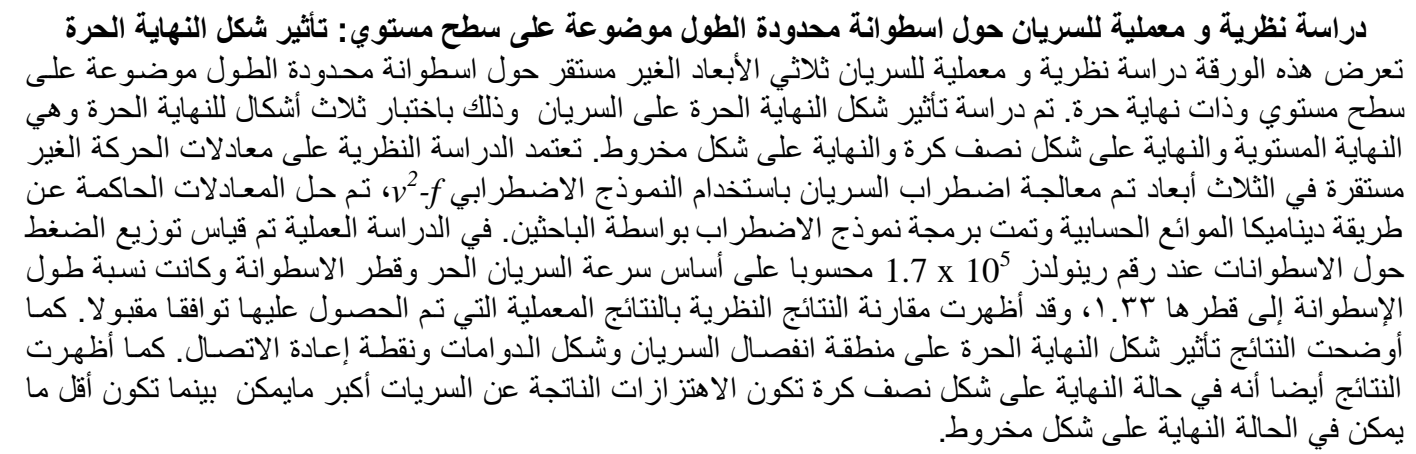

Keywords: bluff body; circular cylinder; vortex shedding, turbulent flow, CFD, unsteady flow

\section{1- INTRODUCTION}

Flow field around bluff bodies such as cars, trains, buildings, stacks, etc. is very complex. Cylindrical geometries are found in many engineering applications such as chimneys, cooling towers, bridge piers, supporters, heat exchangers and car antennas. Therefore, the viscous flow around circular cylinder is the subject of numerous numerical and experimental studies. According to Sumer and Fredsoe [1], flow around circular cylinder begins to separate behind the cylinder at $\mathrm{Re}>40$ causing a vortex shedding which is unsteady phenomenon. The research activities of flow field around cylinders can be divided into two groups: tall cylinders of high aspect ratio, h/D, and short cylinder of low aspect ratio. The unbounded flow around high aspect ratio circular cylinder is a fundamental fluid dynamics problem. The literature in this subject is far extensive to be reviewed here (see for example Coutanceau and Defaye [2], Williamson [3], Catalano et al. [4] and Rahman et al. [5]). The effect of electromagnetic body force on the flow behavior around a circular cylinder was investigated by Kim and Lee [6]. They found that the Lorentz force applied in the circumferential direction on the cylinder moves the separation point rearward, and reduces the drag. The flow around cylinder between two parallel walls was studied by Zovatto and Pedrizzetti [7]. They found that the transition from steady flow to a periodic 
vortex shedding regime has been delayed as the body approaches one wall. Choi and Lee [8] investigated experimentally the flow around an elliptic cylinder located near a flat plate. They found that the variation of surface pressure distributions on the elliptic cylinder with respect to the gap ratio is much smaller than that on the circular cylinder. They found also that the vortices shed regularly only when the gap ratio is greater than the critical value of 0.4 . The critical gap ratio is larger than that of a circular cylinder. Narasimhamurthy et al. [9] studied the flow around tapered cylinder. Their results showed a pattern of discrete oblique shedding cells, which included both vortex dislocation and vortex splitting. Park and Lee [10] investigated experimentally the effects of free-end corner shape on flow structure around a finite cylinder at Reynolds number of 7500. Krajnovic [11] investigated numerically using LES the flow field around a finite circular cylinder with a height-to-diameter ratio of 6 . His results showed that the main recirculation region on the cylinder's top is found to be one vortex with both legs attached to the top surface. The local flow field and near-wake region of a surface-mounted finite circular cylinder were studied experimentally by Rostamy et al. [12, 13]. They found that the point of reattachment of the flow onto the free-end surface moves towards the trailing edge as the cylinder aspect ratio is decreased. The present paper deals the flow around a cylinder of finite length where one end is mounted on a wall and the other is free. This configuration is representative of flows in various applications ranging from buildings, gas tanks and chimneys to flame holders in oil refineries and space rockets on launching pads. The effect of free end shape on the flow field around circular cylinder mounted on flat plate is investigated numerically and experimentally. The cylinder has aspect ratio (length to diameter ratio) of 1.33. Three different free ends are investigated, namely: flat, hemispherical and conical ends. The investigation is performed at Reynolds number of $1.7 \times 10^{5}$ based on free stream velocity and cylinder diameter.

\section{2- MATHEMATICAL MODELING}

The computational fluid dynamics code, Fluent 6.3.26, is used in the present work to solve the Unsteady Reynolds Averaged Navier-Stokes equations (URANS). The numerical method employed is based on the finite volume approach. Fluent provides flexibility in choosing discretization schemes for each governing equation. The discretized equations, along with the initial condition and boundary conditions, are solved using the segregated solution method. Using the segregated solver, the conservation of mass and momentum are solved sequentially and a pressure-correction equation based on the SIMPLE algorithm is used to ensure the conservation of momentum and the conservation of mass (continuity equation).

\subsection{GOVERNING EQUATIONS}

The URANS equations for turbulent incompressible fluid flow with constant properties are used in the present study. The governing flow field equations are the continuity and the Reynolds averaged Navier-Stokes equations, which are given by:

$$
\begin{aligned}
& \frac{\partial}{\partial x_{i}}\left(\rho u_{i}\right)=\mathbf{0} \\
& \frac{\partial}{\partial t}\left(\rho u_{i}\right)+\frac{\partial}{\partial x_{j}}\left(\rho u_{i} u_{j}\right)=-\frac{\partial p}{\partial x_{i}} \\
& +\frac{\partial}{\partial x_{j}}\left[\mu\left(\frac{\partial u_{i}}{\partial x_{j}}+\frac{\partial u_{j}}{\partial x_{i}}\right)\right]+\frac{\partial}{\partial x_{j}}\left(-\rho \overline{u_{i}^{\prime} u_{j}^{\prime}}\right)
\end{aligned}
$$

where, $\overline{u_{i}^{\prime} u_{j}^{\prime}}=\tau_{i j}$ is the unknown turbulent or Reynolds-stress tensor and $u_{i}^{\prime}$ represents the velocity fluctuation in $i$-direction. These equations are not a closed set and turbulence models are required to model the turbulent or Reynolds-stress tensor.

\subsection{TURBULENCE MODELING}

The Reynolds-averaged approach to turbulence modeling requires appropriate modeling of the Reynolds stresses in Eq. (2). A common method employs the Boussinesq hypothesis to relate the Reynolds stresses to the mean velocity gradients:

$$
-\rho \overline{u_{i}^{\prime} u_{j}^{\prime}}=\mu_{t}\left(\frac{\partial u_{i}}{\partial x_{j}}+\frac{\partial u_{j}}{\partial x_{i}}\right)-\frac{\mathbf{2}}{\mathbf{3}} \delta_{i j} \rho k
$$

where, $k=\frac{\mathbf{1}}{\mathbf{2}} \overline{u_{i}^{\prime} u_{j}^{\prime}}$ is the turbulent kinetic energy, $\delta_{i j}$ is the Kronecker delta and $\mu_{t}$ denotes turbulent viscosity. In order to obtain the turbulent viscosity, other transport equations are needed. These equations differ from model to another. Previous investigations [14-16] showed that the $v^{2}-f$ model of Lien and Kalitzin [17] performs better than other models in case of recirculating and separated flows. This model is a simplification of the elliptic relaxation Reynolds stress model developed by Durbin [18], which requires the solution of three transport and one elliptic (relaxation) equations. The system of Reynolds stress equations is replaced by a transport equation for a velocity scalar $\left(\overline{v^{\prime 2}}\right)$ and an elliptic equation for $(f)$. The model was reformulated to avoid the numerical oscillations of wall boundary for $f$, by Lien and Kalitzin [17]. The equations for turbulent kinetic energy and the dissipation rate are the same as those of the standard k- $\varepsilon$ model, while 
the equations for $\overline{v^{\prime 2}}$ and $f$ can be written as given in [17] as follows

The standard $k-\varepsilon$ equations read

$\frac{\partial}{\partial t}(\rho k)+\frac{\partial}{\partial x_{j}}\left(\rho u_{j} k\right)=$

$\frac{\partial}{\partial x_{j}}\left[\left(\mu+\frac{\mu_{t}}{\sigma_{k}}\right) \frac{\partial k}{\partial x_{j}}\right]+\rho\left(P_{r}-\varepsilon\right)$

$\frac{\partial}{\partial t}(\rho \varepsilon)+\frac{\partial}{\partial x_{j}}\left(\rho u_{j} \varepsilon\right)=$

$\frac{\partial}{\partial x_{j}}\left[\left(\mu+\frac{\mu_{t}}{\sigma_{\varepsilon}}\right) \frac{\partial \varepsilon}{\partial x_{j}}\right]+\frac{\rho}{T}\left(C_{1 \varepsilon} P_{r}-C_{2 \varepsilon} \varepsilon\right)$

The $\overline{v^{\prime 2}}$ transport equation is

$\frac{\partial}{\partial t}\left(\rho \overline{v^{\prime 2}}\right)+\frac{\partial}{\partial x_{j}}\left(\rho u_{j} \overline{v^{\prime 2}}\right)=$

$\frac{\partial}{\partial x_{j}}\left[\left(\mu+\frac{\mu_{t}}{\sigma_{k}}\right) \frac{\partial \overline{v^{\prime 2}}}{\partial x_{j}}\right]+\rho\left(k f-\overline{\mathbf{6} v^{\prime 2}} \frac{\varepsilon}{k}\right)$

and the elliptic-relaxation equation $f$ can be represented as:

$L^{2} \frac{\partial^{2} f}{\partial x_{j}^{2}}-f=$

$\frac{\mathbf{1}}{T}\left[\left(C_{\mathbf{1}}-\mathbf{6}\right) \frac{\overline{v^{\prime 2}}}{k}-\frac{\mathbf{2}}{\mathbf{3}}\left(C_{\mathbf{1}}-\mathbf{1}\right)\right]-C_{2} \frac{P_{k}}{k}$

and the turbulent length scale $L$ is determined from the values of $k$ and $\varepsilon$ as follows:

$L=C_{L} \max \left[\frac{k^{3 / 2}}{\varepsilon}, C_{\eta} \frac{(\mu / \rho)^{3 / 4}}{\varepsilon^{1 / 4}}\right]$

The constants of the model are given as follows; see Lien and Kalitzen [17]:

$C_{\mu}=\mathbf{0 . 2 2}, \quad \sigma_{k}=1, \quad \sigma_{\varepsilon}=\mathbf{1 . 3}$,

$C_{1 \varepsilon}=1.4\left(1+0.05 \sqrt{k / \overline{v^{\prime 2}}}\right) ; \quad C_{2 \varepsilon}=1.9$,

$C_{1}=1.4 ; \quad C_{2}=0.3 ; C_{L}=0.23 ; C_{\eta}=70$.

As noticed, all model constants are completely walldistance independent.

The distinguishing feature of the $v^{2}-f$ model is its use of the velocity scale, $\overline{v^{\prime 2}}$ instead of the turbulent kinetic energy, $k$, for evaluating the eddy viscosity. The velocity scale, $\overline{v^{\prime 2}}$ which can be thought of as the velocity fluctuation normal to the streamlines, has shown to provide the right scaling in representing the damping of turbulent transport close to the wall, a feature that $k$ does not provide.

The turbulent viscosity is given by: $\mu_{t}=\rho C_{\mu} \overline{v^{\prime 2}} T$

where $T$ is the turbulent time scale and is given by:

$T=\max \left[\frac{k}{\varepsilon}, 6 \sqrt{\frac{v}{\varepsilon}}\right]$

\subsection{COMPUTATIONAL GRID}

The computational domain used in the present study is $30 \times 30 \times 200 \mathrm{~cm}$ in $\mathrm{x}, \mathrm{y}$ and $\mathrm{z}$ direction respectively. The test cylinder is centered on the lower plate. A structured grid of approximately 1.6 million hexahedral cells is employed in the current work. The grid is clustered toward solid boundaries in order to resolve the boundary layer. The dimensionless wall distance, $y^{+}$is approximately 1 everywhere. The grid is also refined around the cylinder in order to capture the flow structure in that region. Fig. 1 shows the computational grid used in the present study.

\subsection{SOLUTION STRATEGY CONVERGENCE}

AND

A second-order upwind discretization scheme was used for the momentum equation while a first-order upwind discretization was used for turbulent quantities. These schemes ensured, in general, satisfactory accuracy, stability and convergence. Second order discretization is employed for the unsteady term. SIMPLE algorithm described by Patankar [19] was used for pressurevelocity coupling. The discretized equations are solved implicitly in sequence, starting with the pressure equation followed by the momentum equations, by the pressure correction equation, and finally by the equations for the scalars (turbulence variables). Within this loop, the linearized equations for each variable are integrated using a linear system solver. FLUENT code allows implementing customized models through user defined functions, UDF [20]. These capabilities are used to implement $v^{2}-f$ turbulence model. Four user defined scalars, UDS, are used for this purpose. The wall-boundary condition of the dissipation rate equation depends on the turbulent kinetic energy, $\mathrm{k}$, near the wall; therefore, it is necessary to initialize the solution before hooking the boundary condition.

The convergence criterion consists of monitoring cylinder lift coefficient and variation of pressure profiles with flow time. Several time steps are tested and the time step is selected to provide independent results. The results presented hereafter are obtained with time step of $2 \times 10^{-6} \mathrm{sec}$. 


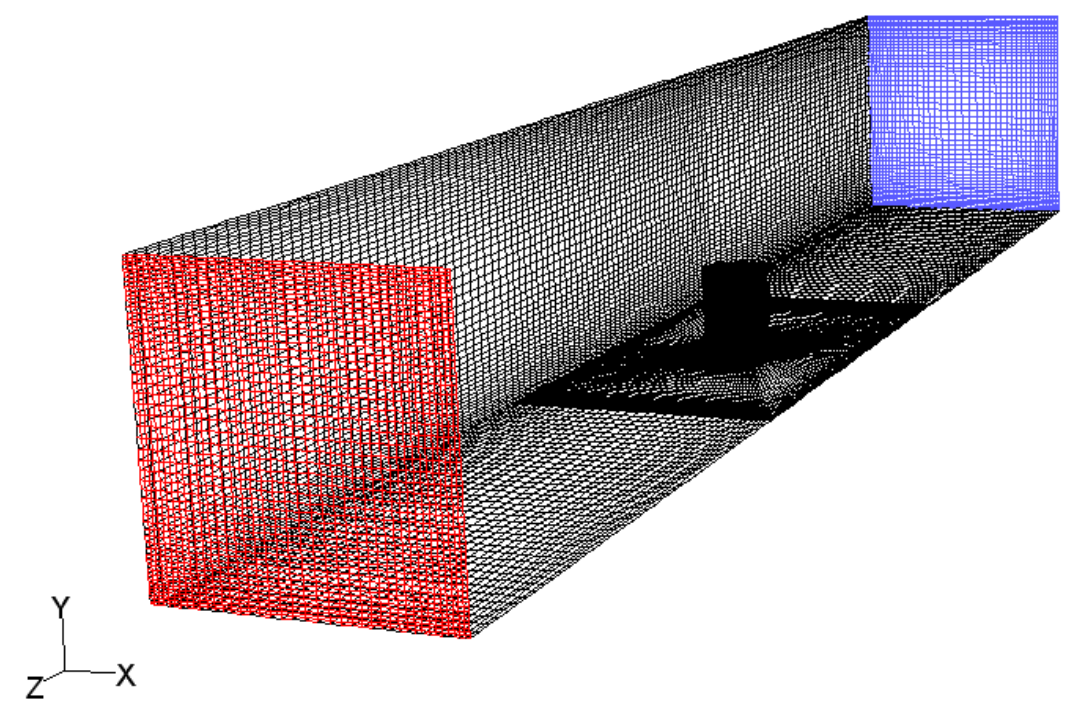

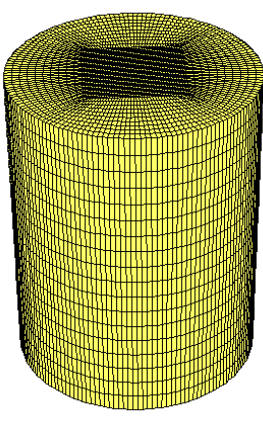

Flat-end

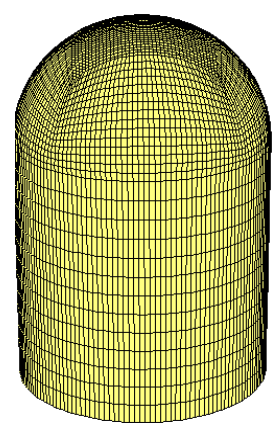

Hemispherical-end

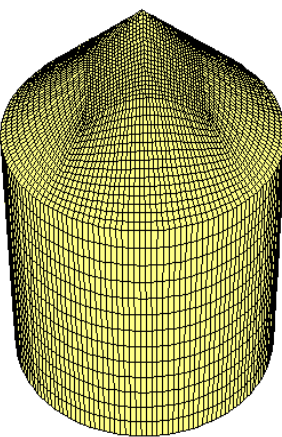

Cone-end

Fig. 1 Details of the computational grid.

\section{3- EXPERIMENTAL STUDY}

Subsonic wind tunnel open and suction type was used to study the flow characteristics around bluff bodies placed on the ground wall. The test section is square cross section $(30 \times 30 \mathrm{~cm})$ and $200 \mathrm{~cm}$. long. In order to ensure that the flow is uniform, the air is drawn into the test section through a regular grid and contraction nozzle which has an area ratio of $1: 10$. The air is exhausted to the atmosphere through a diffuser and a gate valve which is used to control the velocity inside the test section. Figure 2 a shows a schematic diagram of test section. Three models are examined in the present experimental investigations. The models are fabricated from aluminum with 7.5 $\mathrm{cm}$ in diameter and $10 \mathrm{~cm}$ height. The models have different shapes in their top part. It has the shapes of flat top circular-cylinder, hemisphere top cylinder and cone top cylinder, as shown in Figure $2 \mathrm{~b}$. The measurements of the inlet mean velocity is carried out using calibrated pitot-static tube The surface pressure distributions is measured through the pressure tapping holes of $0.5 \mathrm{~mm}$ diameter on the models wall. The model is placed at a downstream distance of $100 \mathrm{~cm}$ from the entrance of the test section. The surface pressure on the models wall is measured for different angle $\left(\theta^{\circ}\right)$ by rotating the model about its vertical y-axis using inclined multitube water manometer. The uncertainty analysis is carried out according to Kline and McClintock method, reported in [21]. It is found that the uncertainties in the measured pressure and velocity are $\pm 0.0098 \mathrm{kPa}$ and $\pm 1.3 \%$, respectively.

\section{4- RESULTS AND DISCUSSION}

The predicted time-averaged pressure coefficient is compared with the measured pressure coefficient at axial position along the tested cylinder in Fig. 3. The pressure coefficient is defined as:

$C_{p}=\frac{p-p_{\infty}}{\mathbf{0 . 5} \rho U_{\infty}^{2}}$

The angle zero degrees in figure 3 corresponds to the upstream stagnation point $(\mathrm{z} / \mathrm{D}=-0.5)$ and increases in the clockwise direction to $180^{\circ}$ at the rear stagnation point $(\mathrm{z} / \mathrm{D}=0.5)$. This figure indicates that 
the agreement between measured and predicted pressure coefficient is acceptable for all cases.

This figure also indicates that the pressure distribution around the cylinder is greatly affected by the free-end shape. Thus, the pressure coefficient at $\mathrm{y} / \mathrm{h}=0.65$ is decreased in the case of hemispherical free-end. This may attributed to the increase of velocity due to streamline curvature in these flow configurations. The figure indicates also that $\mathrm{Cp}$ values decreases as the location of measurements moves towards the free end of the cylinder. This is consistent with the change of the flow around the cylinder due to the flow above the free end and the downwash just behind it.

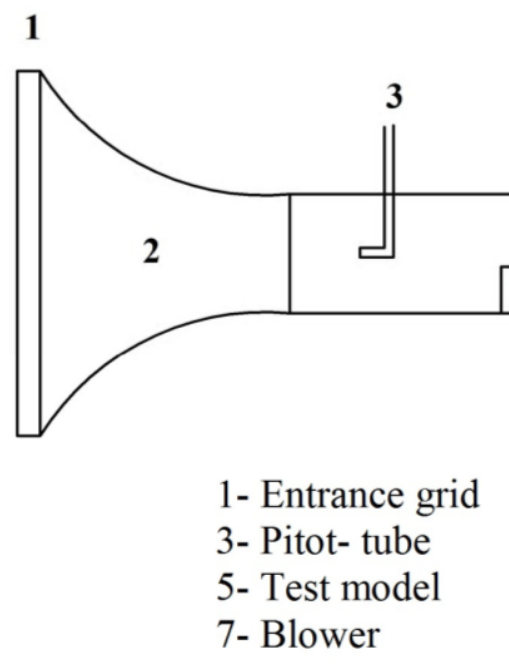

Fig. 2a. The layout of the experimental test rig.
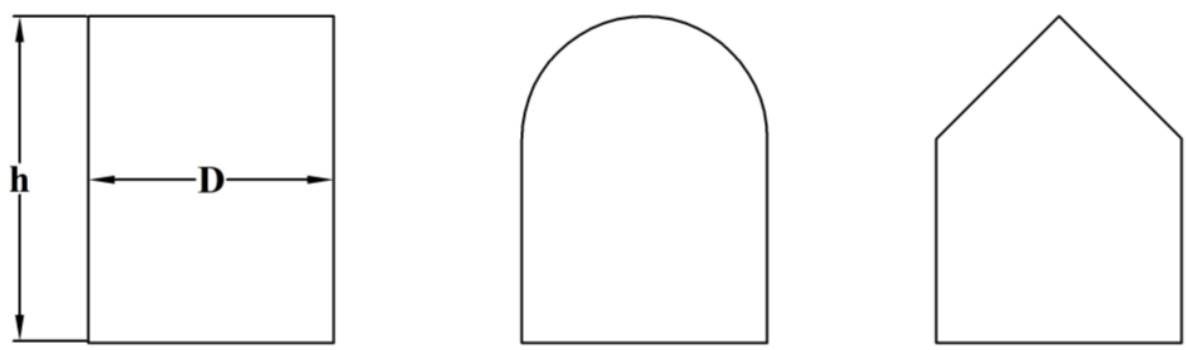

Fig. 2b. The layout of the experimental test rig.

Figure 4 presents the contours of root mean square (RMS) of velocity magnitude for the three tested cylinder. For the case of flat free end cylinder, the incoming flow roll-up from the cylinder circumference and forms a rotating vortex. This kind of vortical flow structure was not observed for the other cylinder models. In addition, the regular vortex shedding on the 3-D cylinder is influenced by the downwash separated flow from the free end. Thus, the hemispherical free end is the most effected by downwash as it has the largest velocity variation. This large variation of velocity downstream of cylinder is shown in Figs. 5 and 6 at two horizontal planes. These figures indicate that the downwash is largest for hemispherical free end. These figures indicate also that the flow downstream of cylinder is nearly symmetric with respect to the central plane.
This is attributed to the fact that the descending shear layer from the free end and the entrained flow from both sides of the finite cylinder are symmetric and they interact in a symmetric manner within the nearwake region. Figure 5 also shows that the wake region downstream of the flat-end cylinder is larger than other cylinders. This can be attributed to the separation bubble formed above the flat free end. The larger velocity variation downstream of hemispherical-end cylinder comes from the fact that smoothed cylinder end concentrates the streamlines which afterwards mixes with the separated shear layer. 


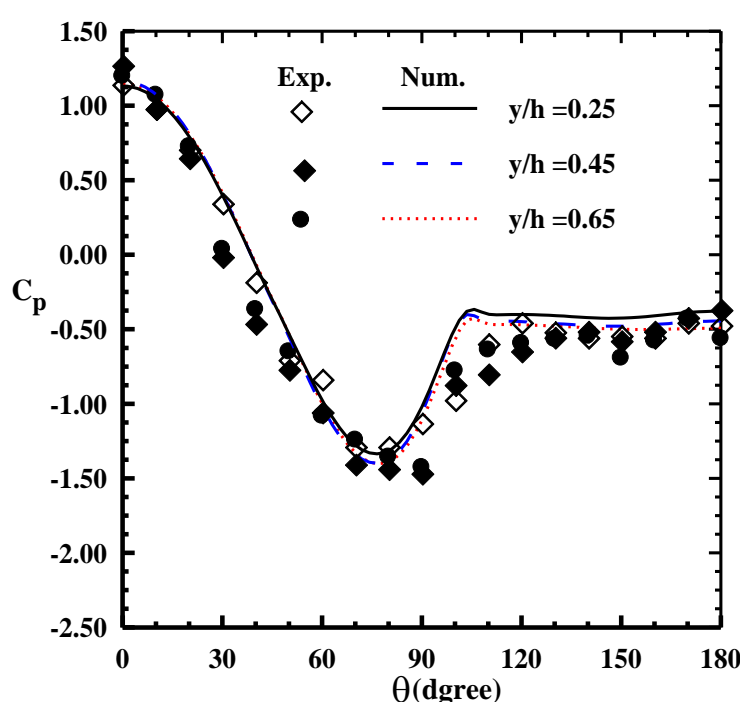

a- Flat-end

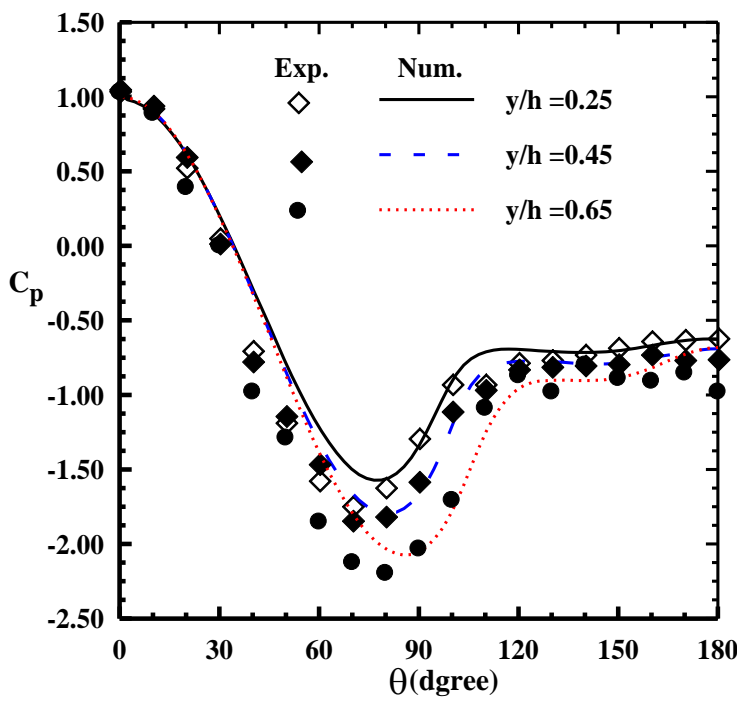

b- Hemispherical-end

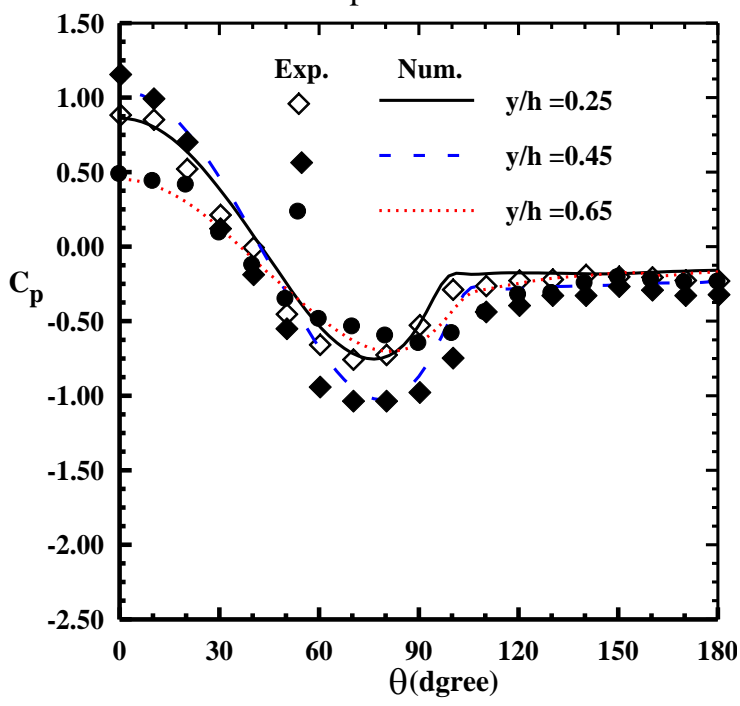

c- Cone-end

Fig. 3. Comparisons between predicted time-averaged pressure coefficient distribution and measured data.
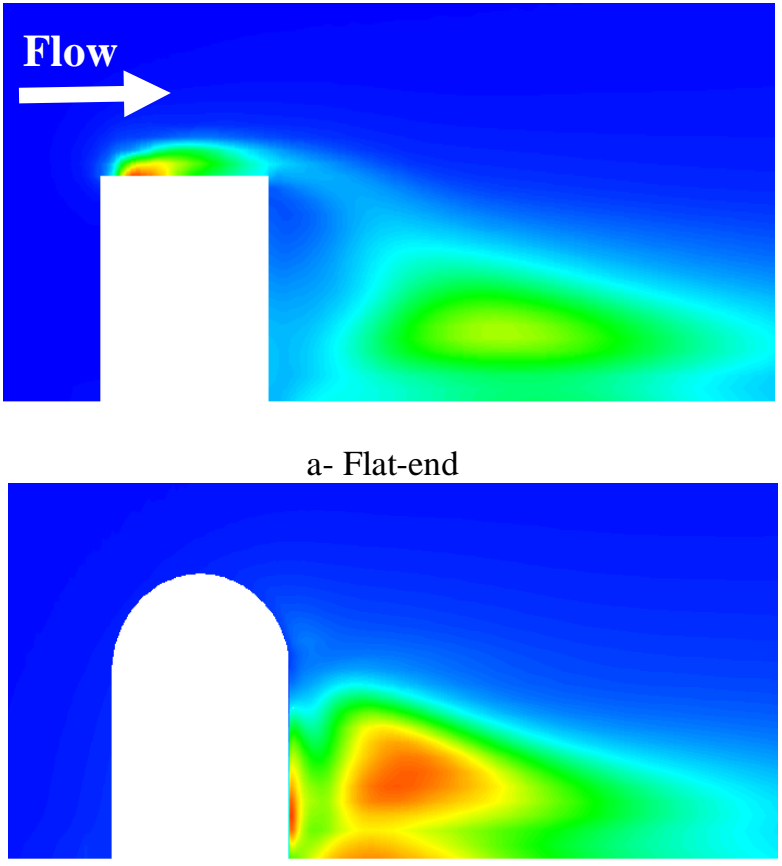

b- Hemispherical-end

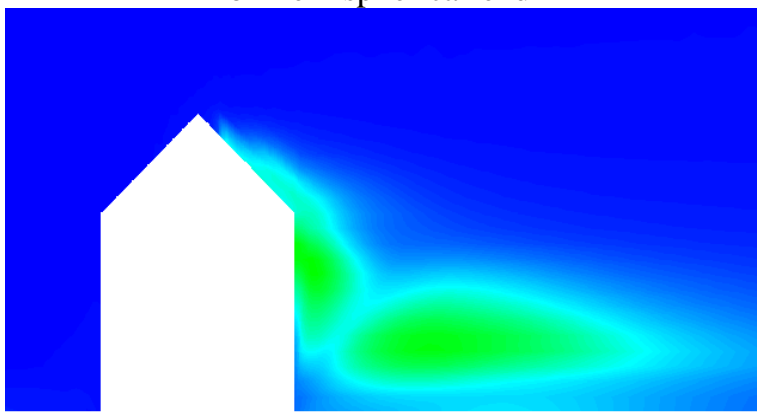

c- Cone-end

$\mathrm{m} / \mathrm{s}$

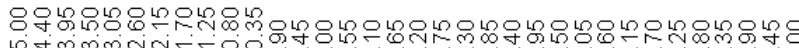

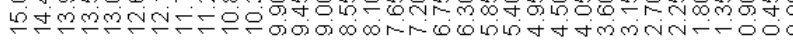

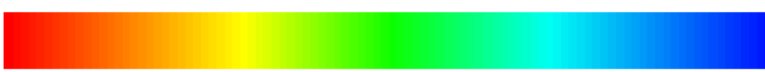

Fig. 4. RMS velocity magnitude in center plan for three tested cylinders (flow from lift to right).

Figures 7 and 8 shows the RMS static pressure at vertical and horizontal planes, respectively. The pressure variation around bluff bodies is the mean reason for flow induced vibration. Due to the large velocity variation of hemispherical-end cylinder, the pressure variation is larger. This pressure variation generates variable lift and drag forces which may produce cylinder vibration. These figures show that the hemispherical free end cylinder has the largest pressure variation and the cone free end is the smallest. Therefore the hemispherical free end cylinder is subjected to the larger flow induced vibration while the cone free end is subjected the lowest flow induced vibration. 


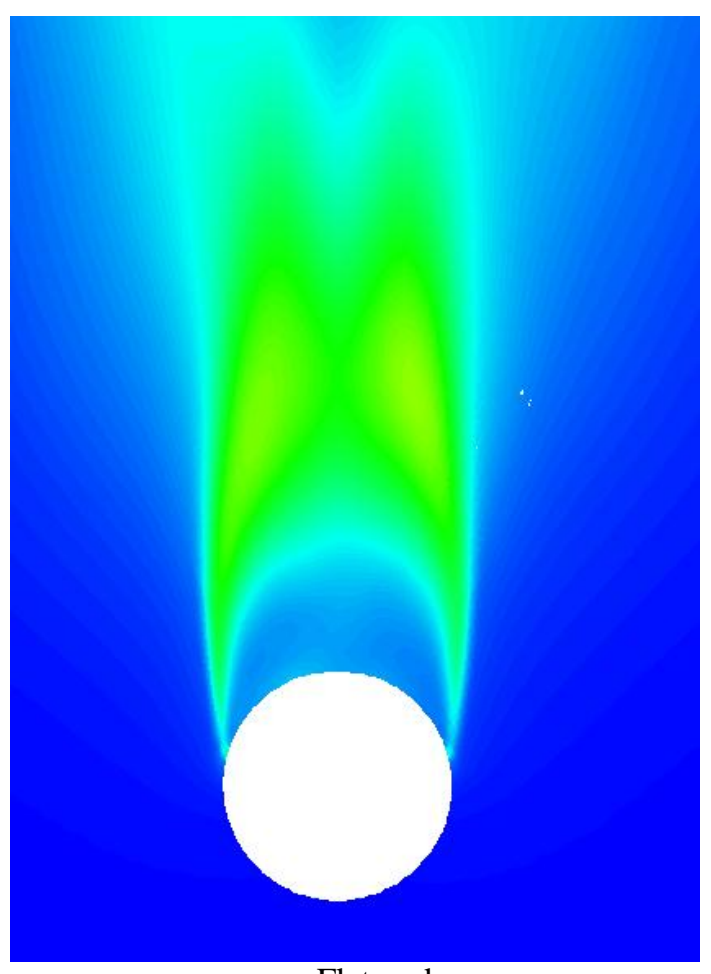

a- Flat-end

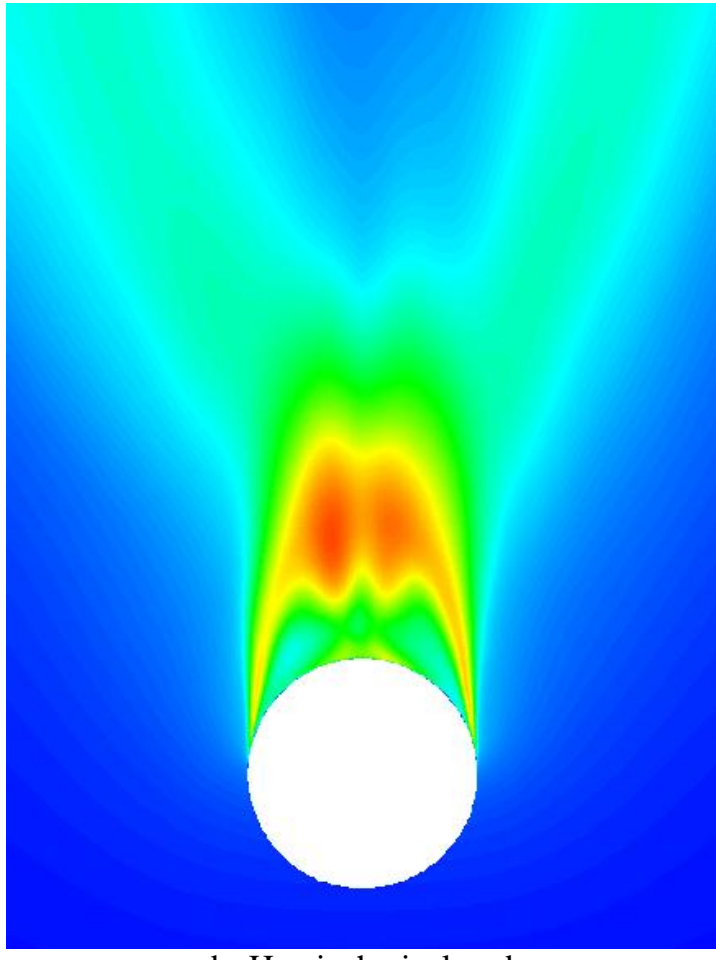

b- Hemispherical-end
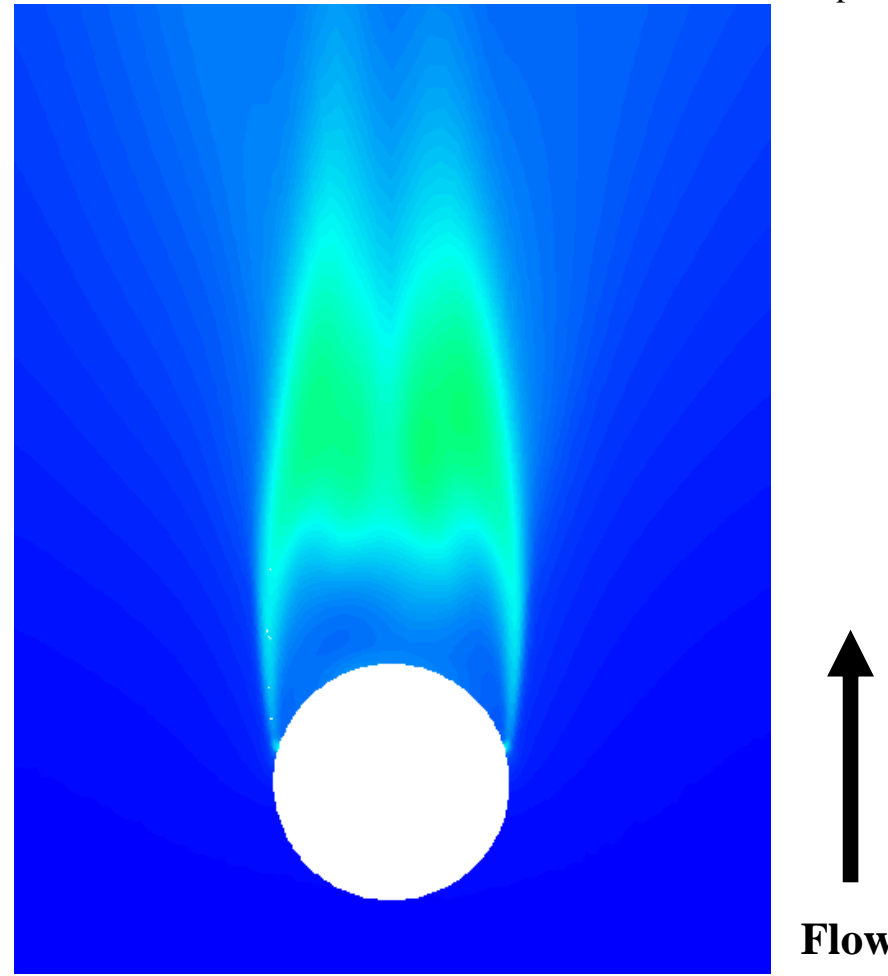

Flow

c- Cone-end

$\mathrm{m} / \mathrm{s}$

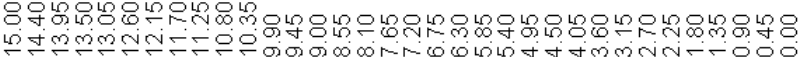

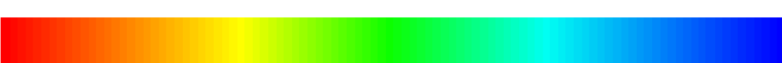

Fig. 5. RMS velocity magnitude in horizontal plan at $y / h=0.5$ for three tested cylinders (flow from Bottom) 


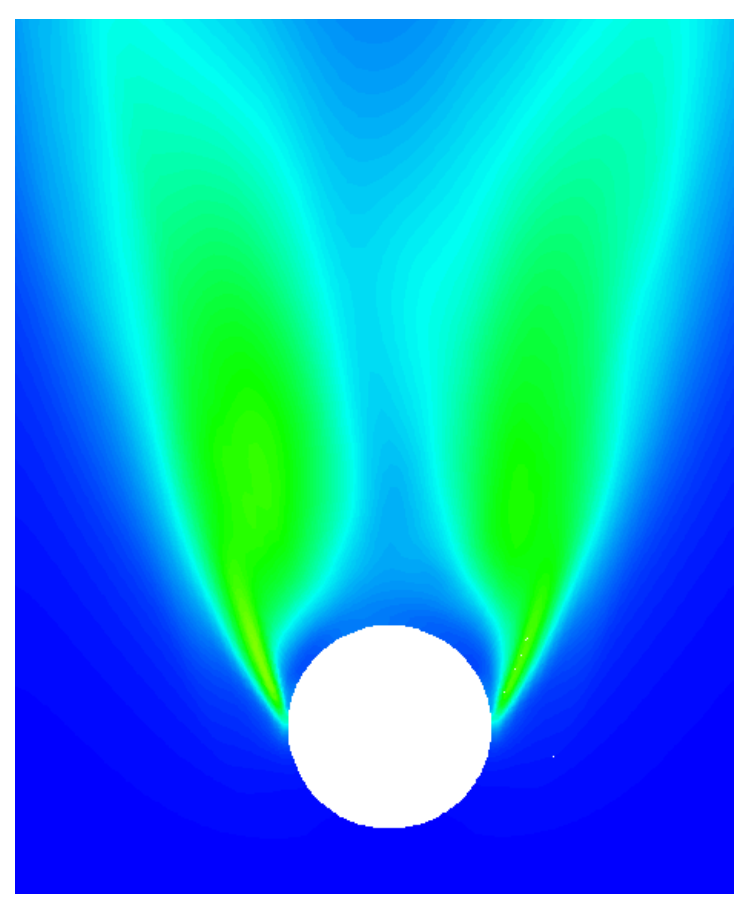

a - Flat-end

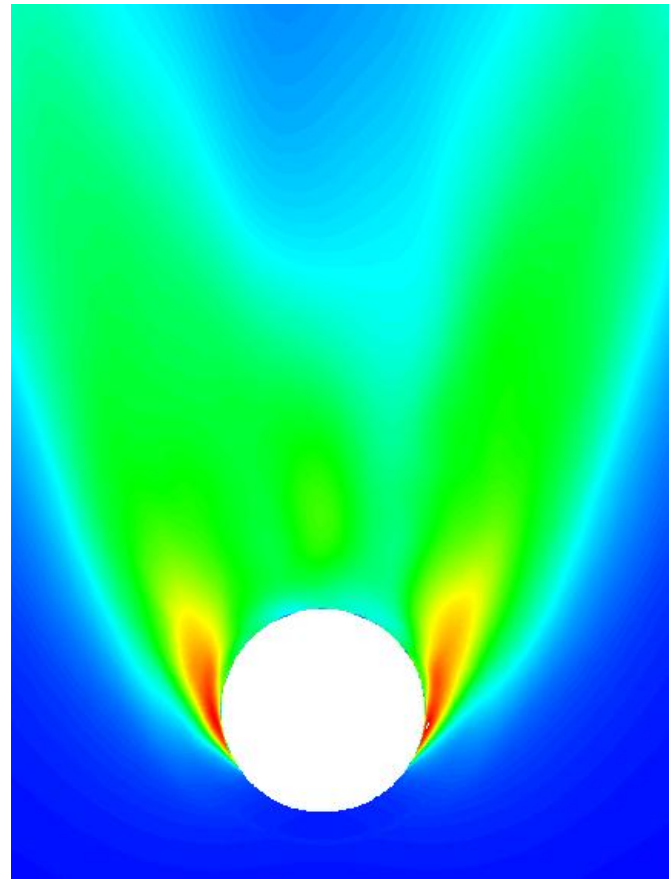

b- Hemispherical-end

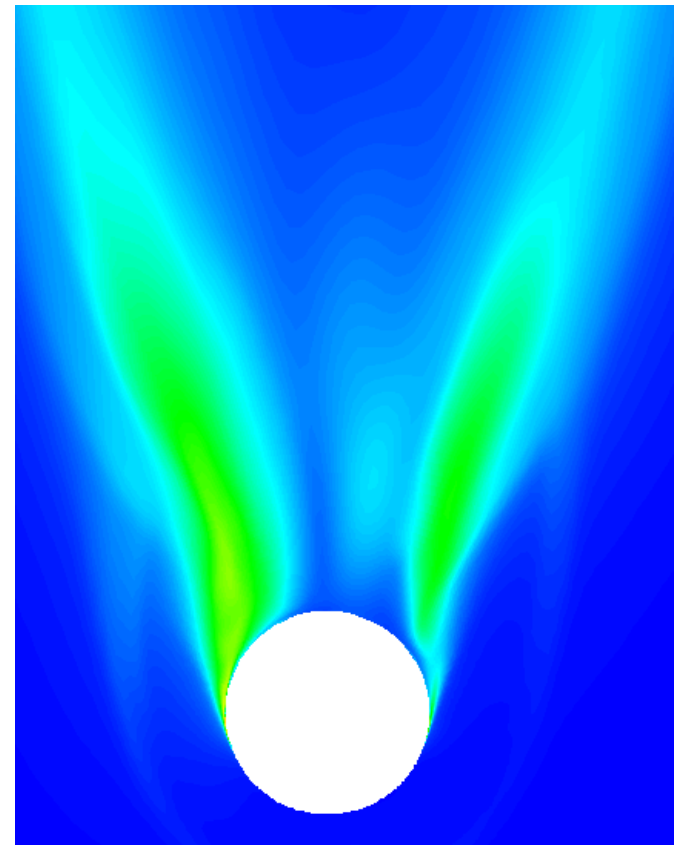

c- Cone-end

$\mathrm{m} / \mathrm{s}$

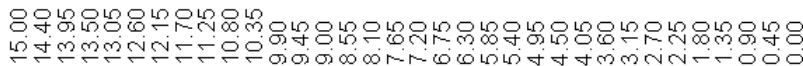

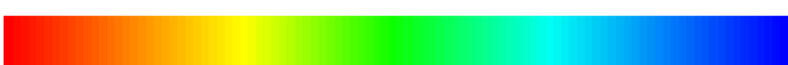

Fig. 6. RMS velocity magnitude in horizontal plan at $y / h=0.0$ for three tested cylinders (flow from Bottom) 


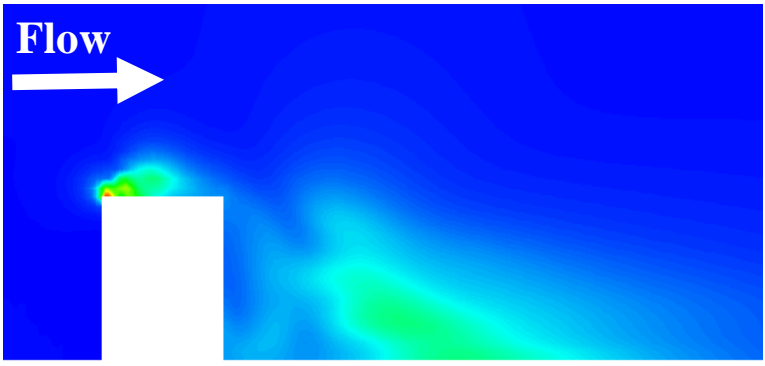

a- Flat-end

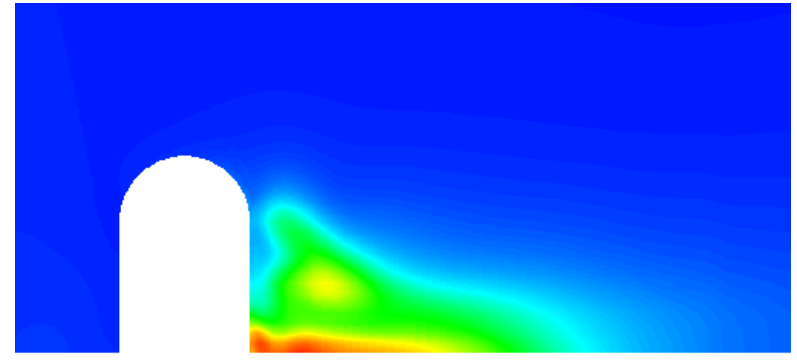

b- Hemispherical-end

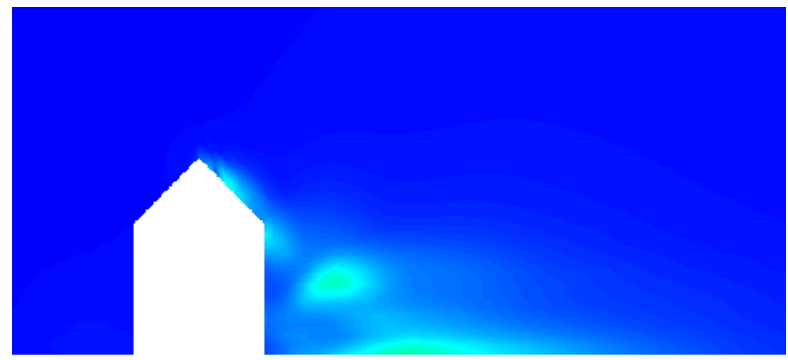

c- Cone-end

$\mathrm{Pa}$

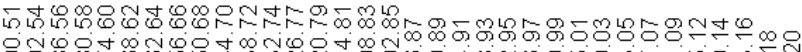

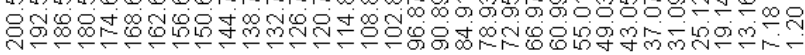

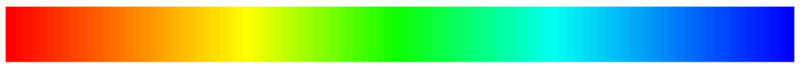

Fig. 7 RMS static pressure in center plan for three tested cylinders (flow from lift to right)

\section{5- CONCLUSIONS:}

The flow field around circular cylinder placed on a flat plate with three different free end shapes was investigated numerically and experimentally. The numerical investigation was carried out using computational fluid dynamic code, fluent. The $v^{2}-f$ turbulence model was implemented in the code using UDF. The surface pressure was measured for the three cylinders of different free ends at Reynolds number of $1.7 \times 10^{5}$ based on the free stream velocity and cylinder diameter. The comparisons between predicted and measured pressure coefficient showed reasonable agreement. The results showed the formation of separation bubble above the flat free end cylinder and the flow downstream the cylinder was nearly symmetric. The results showed also that the wake region downstream of the flat-end cylinder is larger than other cylinders. In addition the hemispherical free end cylinder is subjected to the larger flow induced vibration while the cone free end is subjected to the lowest flow induced vibration.

\section{NOMENCLATURE}

D Cylinder diameter, $\mathrm{m}$

$\mathrm{h} \quad$ Cylinder height, $\mathrm{m}$

$\mathrm{L} \quad$ turbulence length scale, $\mathrm{m}$

$\mathrm{k} \quad$ Turbulent kinetic energy, $\mathrm{m}^{2} / \mathrm{sec}^{2}$

$\mathrm{p} \quad$ Pressure, N/m $\mathrm{m}^{2}$

$\mathrm{P}_{\mathrm{k}} \quad$ Production of turbulence, $\mathrm{kg} / \mathrm{m} / \mathrm{s}^{\mathrm{ec} 2}$

$\mathrm{T} \quad$ turbulence time scale, sec

t Time, sec

$\mathrm{S} \quad$ Strain rate, $1 / \mathrm{sec}$

u Velocity, $\mathrm{m} / \mathrm{sec}$

Greek Symbols

$\varepsilon \quad$ Dissipation rate, $\mathrm{m}^{2} / \mathrm{sec}^{3}$

$\delta \quad$ Kronecker delta

$\mu \quad$ Viscosity, Pa.sec

$\omega \quad$ Specific dissipation rate, $1 / \mathrm{sec}$

$\rho \quad$ Density, $\mathrm{kg} / \mathrm{m}^{3}$

$\tau \quad$ Shear stress, $N / \mathrm{m}^{2}$

Subscripts

in Inlet

t turbulent 


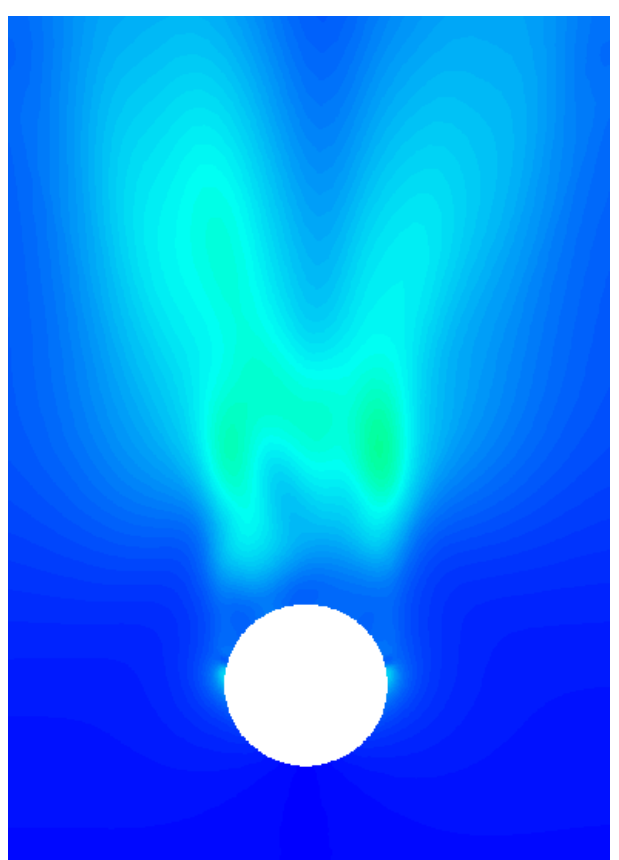

a- Flat-end

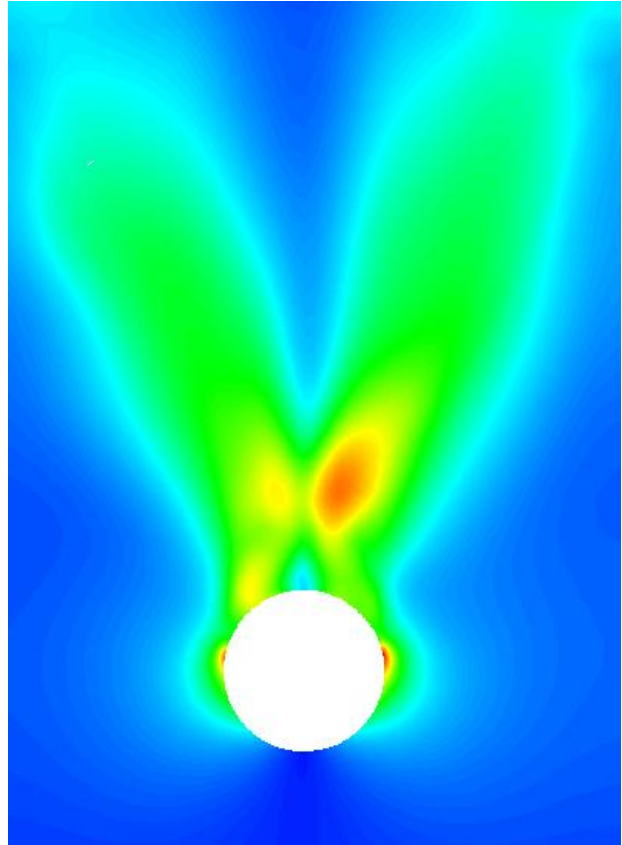

b- Hemispherical-end
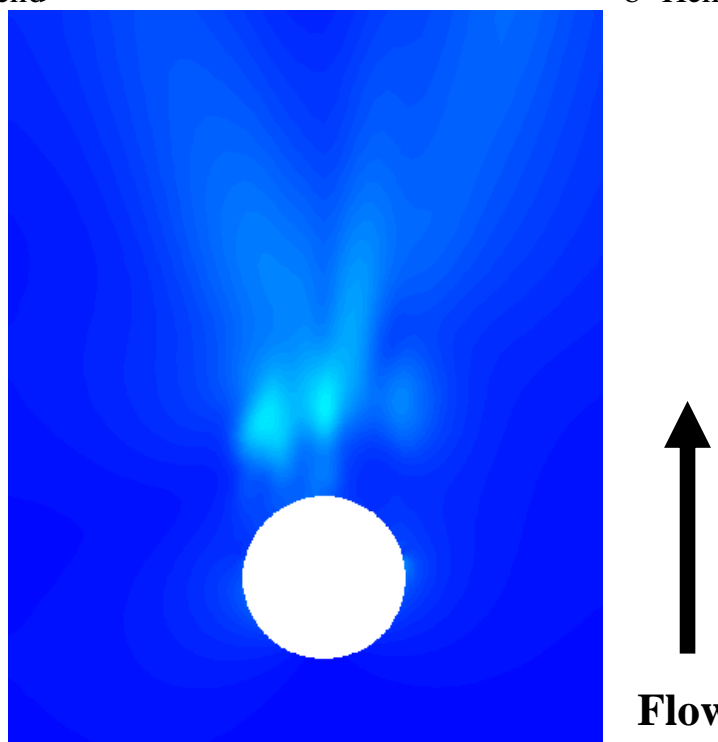

Flow

c- Cone-end

$\mathrm{Pa}$

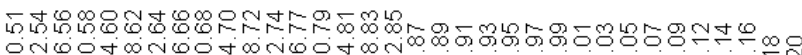

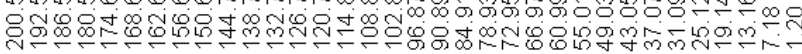

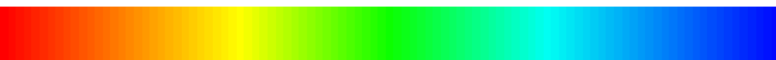

Fig. 8. RMS static pressure in horizontal plan at $y / h=0.5$ for three tested cylinders (flow from Bottom)

\section{REFERENCES}

[1] Sumer, B. M. and Fredsoe J., 1997, "Hydrodynamics around cylindrical structures", World Scientific Publishing Co. Pte Ltd, Singapore.
[2] Coutanceau, M; and Defaye, J.R., 1991, "Circular cylinder wake configurations: a flow visualization survey", Appl Mech Rev., Vol. 44pp. 255-305.

[3] Williamson C.H.K., 1996, "Vortex dynamics in the cylinder wake", Annu. Rev. Fluid Mech., Vol. 28, pp. 477-539. 
[4] Catalano, P., Wang, M., Iaccarino, G., and Moin, P., 2003, "Numerical simulation of the flow around a circular cylinder at high Reynolds numbers", Int. J. Heat and Fluid Flow, Vol. 24, pp.

[5] Rahman, Md. M., Karim, Md. M. and Abdul Alim, Md., 2007, "Numerical investigation of unsteady flow past a circular cylinder using 2-D finite volume method", J. Naval Architecture and Marine Engineering, Vol. 4, pp. 27-42. 463-469.

[6] Kim, S.-J. and Lee, C. M., 2000, "Investigation of the flow around a circular cylinder under the influence of an electromagnetic force", Experiments in Fluids, Vol. 28, pp. 252-260.

[7] Zovatto, L. and Pedrizzetti, G., 2001, "Flow about a circular cylinder between parallel walls", J. Fluid Mech., Vol. 440, pp. 1-25.

[8] Choi, J.-H. and Lee, S.-J, 2000, "Ground effect of flow around an elliptic cylinder in a turbulent boundary layer", Journal of Fluids and Structures, Vol. 14, pp. 697-709.

[9] Narasimhamurthy, V.D., Schwertfirm, F., Andersson, H.I. and Pettersen, B., 2006, "Simulation of unsteady flow past tapered circular cylinders using an immersed boundary method", European conference on computational fluid dynamics, Wesseling, P. Onate E., and j. Periaux J. (Eds.), TU Delft, the Netherlands.

[10] Park, C.W. and Lee, S.J., 2004, "Effects of freeend corner shape on flow structure around a finite cylinder", Journal of Fluids and Structures, Vol. 19 pp.141-158.

[11] Krajnovic, S., 2011, "Flow around a tall finite cylinder explored by large eddy simulation", J. Fluid Mech., Vol. 676, pp. 294-317.

[12] Rostamy, N., Sumner, D., Bergstrom, D.J., Bugg, J.D., 2012, "Local flow field of a surfacemounted finite circular cylinder", Journal of Fluids and Structures, Vol. 34, pp. 105-122.
[13] Rostamy, N., Sumner, D., Bergstrom, D.J., Bugg, J.D., 2013, "Instantaneous flow field above the free end of finite-height cylinders and prisms" Int. J. of heat and fluid flow, in press.

[14] El-Askary W.A., Balabel A., El-Behery S.M., Hegab A., 2011 ,"Computations of a Compressible Turbulent Flow in a Rocket Motor-Chamber Configuration with Symmetric and Asymmetric Injection", Computer Modeling in Engineering \& Sciences, Vol. 82, pp.29-54.

[15] El-Behery S. M., Hamed M. H., 2011, "A Comparative Study of Turbulence Models Performance for Turbulent Flow in A Plane Asymmetric Diffuser", Computers and Fluids, Vol. 44, pp. 248-257.

[16] Balabel A, Hegab A., Nasr M. and El-Behery S. M., 2011, "Assessment of Turbulence Modeling for Gas Flow in Two-Dimensional ConvergentDivergent Rocket Nozzle" Applied Mathematical Modelling, Vol. 35, pp. 3408-3422.

[17] Lien F-S and Kalitzin G, 2001, "Computations of transonic flow with the $v^{2}-f$ turbulence model", Int. J. Heat Fluid Flow, Vol. 22, pp. 53-61.

[18] Durbin P. A., 1991, "Near-wall turbulence closure modeling without damping functions", Theoretical and Computational. Fluid Dynamics, Vol. 3, pp. 1-13.

[19] Patankar S. V., 1983, "Numerical heat transfer and fluid flow", McGraw-Hill, New York, USA.

[20] Fluent, 2006, "User's Guide Fluent 6.3.26", Fluent Incorporated, Lebanon, NH.

[21] Holman, J.P., 2000, "Experimental methods for engineers", 7th Ed., McGraw-Hill. 